\title{
Study On the Mechanism of LMP2A Maintaining Epstein-barr Virus Latency Infection Through Interaction With CXCR4
}

Ni Qin

Qingdao University Medical College

Yan Zhang

Zibo Central Hospital

Lin Xu

Qingdao Municipal Hospital Group

Wen Liu

Qingdao University Medical College

Bing Luo ( $\nabla$ qdluobing@163.com )

Qingdao University Medical College https://orcid.org/0000-0002-6541-5958

\section{Research Article}

Keywords: Epstein-Barr virus, LMP2A, EBNA1, CXCR4

Posted Date: November 29th, 2021

DOI: https://doi.org/10.21203/rs.3.rs-1088199/v1

License: (c) (1) This work is licensed under a Creative Commons Attribution 4.0 International License. Read Full License 
3 Ni Qin ${ }^{1}$, Yan Zhang ${ }^{1,2}$, Lin Xu ${ }^{3}$, Wen Liu ${ }^{1}$, Bing Luo ${ }^{1, *}$

4 1. Department of Pathogeny Biology, Basic Medicine College, Qingdao University, Qingdao 5 266071, China.

6 2. Department of Clinical Laboratory, Zibo Central Hospital, Zibo 255000, China.

7 3. Department of Gastroenterology, Qingdao Municipal Hospital, 5 Donghai Middle Road, 8 Qingdao, 266000, China.

9 *Corresponding author: Bing Luo, Department of Pathogeny Biology, Basic Medicine College,

10 Qingdao University, No.308 Ningxia Road, Qingdao 266071, China. Phone: 86-32-82991083; E-

11 mail: qdluobing@163.com

12 Keywords: Epstein-Barr virus, LMP2A, EBNA1, CXCR4 


\section{Abstract}

Epstein-Barr virus (EBV) belongs to the $\gamma$-herpesvirus subfamily and is the first human tumor

30 virus to be discovered. The global adult infection rate exceeds $90 \%$. EBV can participate in the

31 regulation of multiple genes and multiple signal pathways through its latent genes. Many studies

32 have reported that CXCR4 is involved in the development of gastric cancer, but there are few studies

33 on the specific mechanism of its role in EBV-associated gastric cancer (EBVaGC). In this study, we

34 explored the mechanism by which EBV-encoded products maintain EBV latent infection through

35 interaction with CXCR4, and the role of CXCR4 in EBV positive cells. The results show that there

36 is a positive feedback between the EBV-encoded products and CXCR4, and LMP2A can activate

37 CXCR4 through the NF-kB pathway. In addition, CXCR4 can be fed back to LMP2A and EBNA1

38 through the ERK signaling pathway. At the same time, CXCR4 can promote the proliferation and

39 migration of EBV-positive cells, reduce the expression of the immediate early protein BZLF1, and

40 play an important role in maintaining the incubation period of EBV infection. These findings are

41 conducive to the further targeted therapy of EBVaGC. 


\section{Introduction}

Epstein-barr virus (EBV) belongs to the $\gamma$-herpesvirus subfamily, and the infection rate of adults in the world is more than $90 \%$. EBV is a widely recognized tumor-associated virus [34], which is closely related to the occurrence and development of various tumors. Most EBV-related tumors are caused by the virus establishing a long-term latent infection in host cells after the initial lytic infection. EBV has two modes of infection: latent infection and lytic infection. The latent infection status of EBV is an important carcinogenic basis. According to the expression of EBNA and LMP [28], EBV shows three different types of latent infection, while EBV-associated gastric cancer (EBVaGC) belongs to type I latent cancer, mainly expressing LMP2A and EBNA1 [21]. In addition, BZLF1 is the earliest expression protein of EBV genome after activation from latent state, and the transcriptional activation function of this gene plays a key role in viral gene from latent activation to proliferation [25].

C-X-C motif chemokine receptor 4 (CXCR4), a 352 amino acid rhodopsin like GPCR, was first studied as a chemokine receptor associated with breast cancer metastasis to lung tissue [30]. CXCR4 is the most widely expressed chemokine receptor in more than 23 human cancers, including breast cancer, ovarian cancer, melanoma, etc., and is involved in tumor growth, angiogenesis, metastasis, etc. [4]. Several studies have reported that CXCR4 is involved in the proliferation and metastasis of gastric cancer and is closely related to the clinical prognosis of gastric cancer [31, 32]. EBVaGC is a unique molecular subtype of gastric cancer that accounts for $8.7 \%$ of the total number of gastric cancers according to big data [20]. Based on the high incidence and mortality of gastric cancer worldwide, the pathogenesis of EBVaGC is important.

EBV can participate in the regulation of multiple signaling pathways through its encoded latent genes, such as NF- $\mathrm{B}, \mathrm{JNK}, \mathrm{PI} 3 \mathrm{~K} / \mathrm{AKT}$, etc., and then regulate the expression of downstream target genes to promote the occurrence and development of tumors. Longnecker et al. found that LMP2A could simulate $\mathrm{B}$ cell receptor (BCR) signaling and regulate the activation of related signaling pathways through its cytoplasmic n-terminal region [16]. In EBVaGC, LMP2A activates NF- $\mathrm{B}$ signaling by upregulating Survivin expression [7]. CXCR4-ERK signaling pathway mediates 
82 proliferation, migration, and invasion of cancer cells in multiple types of tumors [2, 15, 18]. However,

83 the specific mechanisms by which CXCR4 plays a role in EBVaGC are poorly studied. The purpose

84 of this study was to investigate the mechanism of interaction between CXCR4 and EBV coding products and the involvement of CXCR4 in maintaining EBV latent infection.

\section{Materials and Methods}

\subsection{Cell lines and culture conditions}

In this study, SNU719 and GT38 cell lines were EBV-positive gastric epithelial cell lines. SNU719 cells were provided by Professor Qian Tao (The Chinese University of Hong Kong), expressing LMP2A and EBNA1. GT38 cells were kind gifts by Sairenji T (Tottori University, Japan) and expressed LMP1, EBNA1 and EBNA2. SGC7901, an EBV-negative gastric epithelial cell line, was purchased from the Cell Bank of the Chinese Academy of Sciences. All cell lines were cultured in DMEM (Gibco, USA) supplemented with 10\% fetal bovine serum (Biological Industries, Israel) and $2 \%$ penicillin-streptomycin, at $37{ }^{\circ} \mathrm{C}$ with $5 \% \mathrm{CO}_{2}$.

\subsection{RNA isolation and Quantitative Real-time PCR(qRT-PCR)}

Total RNA was extracted from cell lines with TRIzol reagent (Invitrogen, USA) and reverse transcribed using First Strand cDNA synthesis kit (Takara, Japan), according to the manufacturer's instructions. These products were using a FastStart DNA Master SYBR Green Kit (Roche, Germany) in the Light Cycler 96 sequence detection system. All reactions were done in triplicate and relative gene expression was calculated using the comparative cycle threshold $(\mathrm{Ct})$ value $\left(2^{-\Delta \Delta \mathrm{Ct}}\right)$, using $\beta$ actin as the internal standard. The sequences of the specific forward and reverse primers were as

LMP2A, 5'-TGTCGCTGGCATACTCTTCA-3', and $5^{\prime}-$

103 GCGTGTTAGTCATCACCGTC-3', for $\beta$-actin $5^{\prime}$-TCCTGTGGCATCCACGAAACT-3', and 5'GAAGCATTTGCGGTGGACGAT-3'.

\subsection{Western blotting analysis}

All cells were washed twice with cold phosphate buffer solution (PBS) and lysed in RIPA buffer mixture (RIPA : PMSF : phosphatase inhibitors, $100: 1: 1$ ), and then mixed with loading buffer 

device. The blotted PVDF membranes were blocked with 5\% non-fat milk for $2 \mathrm{~h}$ at room temperature and incubated with the primary antibody at $4{ }^{\circ} \mathrm{C}$ overnight, followed by a second antibody for incubating $2 \mathrm{~h}$. Finally, proteins of interest were visualized using the enhanced chemiluminescence detection system. The specific antibodies used were as follows: Anti- $\beta$-actin,

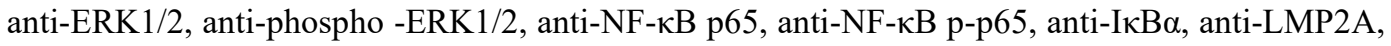
anti-MMP-2, anti-Vimentin, HRP-linked anti-mouse/rabbit secondary antibody .All above antibodies were purchased from CST (Mass, USA), at a dilution of $1: 1000$. Anti-CXCR4, anti-Ecadherin, anti-N-cadherin antibodies were purchased from Abcam (USA) with a dilution of $1: 1000$.

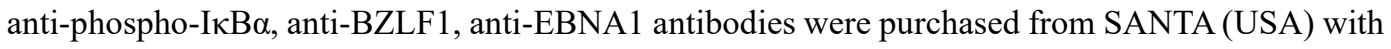
a dilution of $1: 200$.

\subsection{Transfection with siRNAs of LMP2A、CXCR4 and ERK}

The siRNA target sequences for LMP2A, CXCR4 and ERK mRNA were designed and synthesized by GenePharma (China). The sequences are as follows: hs-CXCR4-si-1: 5' AGAUAUACACUUCAGAUAAdTd - 3', 5' - UUAUCUGAAGUGUAUA

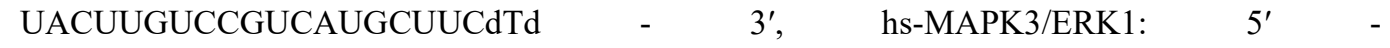

\subsection{CCK-8 cell proliferation assay}

Cell proliferation was detected using the Cell Counting Kit 8 (CCK8, Boster Biological 
136 Technology, China). The cells were seeded at $5 \times 10^{3}$ cells $/ 100 \mu 1$ per well into 96 -well plates. After

137 the cells were attached to the wall, added $10 \mu \mathrm{lCCK}-8$ solution to each well, after incubation for 24 ,

13848,72 and 96h, respectively. And then the cells were incubated for an additional $1 \mathrm{~h}$ at $37^{\circ} \mathrm{C}$, the

139 absorbance was measured using Soft-Max apparatus (Bio-Tek ELx808, USA) at a wavelength of

$140 \quad 450 \mathrm{~nm}$.

$141 \quad 2.6$ Migration assay

142 In the transwell migration assay, the transwell chamber was topped with a non-coated 143 membrane with $8 \mu \mathrm{m}$ pores (Corning, USA), to which a serum-free culture medium containing $1441 \times 10^{5}$ cells were added, and to which $20 \%$ fetal bovine serum was added as a chemical attractant in 145 the lower chamber. After incubation for $24 \mathrm{~h}$, the cells were fixed with methanol, stained with 146 hematoxylin, and the stained cells were counted under $200 \times$ magnification under a microscope. All 147 experiments had three independent repetitions.

\section{$148 \quad 2.7$ Overexpression of LMP2A and CXCR4}

149 The plasmids of LMP2A (pcDNA3.1-LMP2A) and CXCR4 (pcDNA3.1-CXCR4) were 150 purchased from Invitrogen Inc. (Hanbio, China) and were transfected using Lipofectamine 2000 151 Reagent (Invitrogen, USA). Fluorescent activity was measured under a fluorescence microscope. 152 SGC7901 cells and SNU719 cells were seeded at $1 \times 106$ cells per well in 6 -well plates and 153 transfected with $2.5 \mu \mathrm{g}$ LMP2A, CXCR4 or negative control vector plasmids. Stable transfections of 154 LMP2A and CXCR4 were selected by G418. After successful transfection and screening, cells were 155 collected for experiments.

$156 \quad 2.8$ Statistical analysis

157 Data were analyzed with using Student's t-test. Analyses were performed with using GraphPad 158 Prism software (GraphPad Software, USA). Differences were considered statistically significant if $159 P<0.05$. All Data was expressed as means \pm standard error of the mean (SEM) and all experiments 160 were repeated at least three times.

1613 Results

$162 \quad 3.1$ Interaction between LMP2A and CXCR4 at the expression level 
EBV-positive gastric cancer cell line SNU719 was stably transfected with CXCR4 gene and EBV-negative gastric cancer cell line SGC7901 was stably transfected with LMP2A gene. Fig.1a showed the transfection efficiency of the plasmid in cells. The corresponding mRNA and protein expression was detected by qRT-PCR and Western blot, Fig.1b showed that the expression of LMP2A increased in SGC7901 cells after transfection with LMP2A (SGC7901-LMP2A) compared with SGC7901-NC cells. Fig.1c showed that the expression of CXCR4 in SGC7901-LMP2A cells was higher than that in SGC7901-NC cells. Meanwhile, after treatment with LMP2A siRNA, the expression of LMP2A was significantly decreased in GT38 cells, and the expression of CXCR4 was down-regulated at the protein level (Fig.1d). Fig.1e showed the up-regulated expression of LMP2A and EBNA1 in SNU719-CXCR4 cell line that stably expressed CXCR4. In addition, Western blot was used to detect the expression of LMP2A and EBNA1 related proteins after CXCR4 siRNA treatment (Fig.1f). The results showed that CXCR4 siRNA significantly reduced the expression of LMP2A and EBNA1 in SNU719 cells compared with the control group.

\subsection{LMP2A regulates CXCR4 expression through the NFKB pathway}

Data showed that CXCR4 protein expression increased in SGC7901-LMP2A cells with stable LMP2A expression. We intend to further explore the specific ways in which LMP2A regulates CXCR4. In a follow-up validation experiment, we found higher expression levels of NF- $\mathrm{KB}$ pathway related protein p65 and phosphorylated p65(p-p65) in SGC7901 compared with the control group (Fig.2a). Meanwhile, the expression of p65 and p-p65 in GT38 cells was significantly decreased after LMP2A siRNAs treatment (Fig.2b). In order to make the experimental results more comprehensive and accurate, the concentrations of $2 \mu \mathrm{M}$ and $4 \mu \mathrm{M}$ of NF- $\kappa \mathrm{B}$ inhibitor BAY-7085 were cultured in six-well plates inoculated with GT38 cells for 24h, respectively. Western blot results showed that NF-KB pathway related proteins were significantly reduced in the inhibitor group and showed a dose- dependent manner (Fig.2c). Similarly, when $2 \mu \mathrm{M}$ and $4 \mu \mathrm{M}$ NF- $\mathrm{kB}$ inhibitor BAY-7085 were added to SNU719 cells, NF-אB pathway related proteins were significantly reduced in the inhibitor group, also showing a dose-dependent manner (Fig.2d).

\subsection{CXCR4regulates LMP2A and EBNA1 expression through the ERK pathway}


In SNU719-CXCR4 cells that stably expressed CXCR4, the protein expressions of LMP2A and EBNA1 were increased. It was further found that CXCR4 overexpression induced the expression of ERK and phosphorylated ERK(p-ERK) (Fig.3a). Meanwhile, after CXCR4 siRNA treatment, the expressions of ERK and p-ERK in GT38 and SNU719 cells were significantly decreased (Fig.3b, Fig.3c). In order to further understand the mechanism of CXCR4 overexpression leading to the up-regulation of LMP2 and EBNA1, the ERK1/2 inhibitor PD0325901 at $2 \mu \mathrm{M}$ and $4 \mu \mathrm{M}$ concentrations was cultured in six-well plates inoculated with GT38 cells for $48 \mathrm{~h}$, respectively. Western-blot results showed that p-ERK was significantly reduced in the inhibitor treatment group, and LMP2A and EBNA1 were also reduced to different degrees in a dose-dependent trend (Fig.4a). Similarly, $2 \mu \mathrm{M}$ and $4 \mu \mathrm{M}$ ERK1/2 inhibitor PD0325901 were added to SNU719 cells, respectively, with the same results as GT38 cell line (Fig.4b). In addition, we found that knockdown ERK1/2 had a strong inhibitory effect on the expression of LMP2A and EBNA1 (Fig.4c, Fig.4d). In conclusion, these results suggest that LMP2A and EBNA1 expression in EBVaGC is partially dependent on the CXCR4-ERK pathway.

\subsection{Effects of CXCR4 on cell migration, proliferation and maintenance of EBV latent} infection

CXCR4 was overexpressed in SNU719 to explore the effect of CXCR4 on cell phenotypic function. The effect of CXCR4 on the migration ability of SNU719 cells was detected by Transwell migration experiment. Fig.5a showed that the overexpression of CXCR4 significantly promoted the migration of SNU719-CXCR4 cells compared with SNU719-NC cells. CCK-8 detection showed that CXCR4 overexpression significantly increased the OD value of SNU719 cells (Fig.5b). In addition, the Western blot results showed that compared with the control group, the protein expressions of EMT-related proteins N-Cadherin, MMP-2, and Vimentin in SN719-CXCR4 were

213 increased, while the expression of epithelial Cadherin E-Cadherin was decreased. (Fig.5c). In 214 addition, we further investigated the effect of CXCR4 expression on the latency of EBV based on 215 the discovery that CXCR4 promotes LMP2A and EBNA1, the latent genes encoded by EBV. 216 SNU719 cells were treated with DMSO, DMSO+ transfected CXCR4, TPA and TPA+ transfected 
217 CXCR4 respectively. The expression of BZLF1 in TPA treated cells was significantly higher than

218 that in the other three groups. Decreased BZLF1 expression was observed in TPA+ transfected

219 CXCR4 group. In addition, the expression of BZLF1 in DMSO+ transfected CXCR4 group was

220 lower than that in the other three groups (Fig.5d). These data suggest that CXCR4 can inhibit the

221 expression of BZLF1 and promote the expression of LMP2A and EBNA1, the latent genes encoding

222 EBV. In conclusion, CXCR4 promotes EBVaGC cell migration and proliferation, and may play an

223 important role in maintaining EBV latent infection during EBVaGC development.

\section{Discussion}

225 CXCR4 is a seven-transmembrane G-protein-coupled receptor, with chemokine 12 (CXCL12) 226 as its typical ligand [11]. CXCR4 is highly expressed in various types of tumor cells and tissues, and 227 can be involved in tumor growth, invasion, angiogenesis and metastasis, such as breast cancer, 228 colorectal cancer, pancreatic cancer, etc. [13, 17, 23]. Studies have shown that CXCR4 is regulated 229 by a variety of factors, including its activation by binding to the chemokine CXCL12, and activation 230 by macrophage migration inhibitory factor (MIF) and extracellular ubiquittin [1, 22]. In addition, 231 EBV-encoded products can also regulate the expression of CXCR4. Huo et al. found that EBNA1, 232 as a tumor promoter, enhanced the chemoinductance of Treg cells by targeting CXCL12-CXCR4 to 233 promote NPC immune escape [9]. In Xu's study, the results showed that in NPC, LMP1 affects cell 234 motility and invasion by inducing CXCR4 [33]. According to the different expression patterns of 235 EBV protein, EBVaGC was classified as latent type I or II, mainly expressing EBER, EBNA1, 236 LMP2A and other latent products [6]. In this study, CXCR4 expression increased or decreased in 237 EBVaGC cells as LMP2A was overexpressed or knocked down. In addition, CXCR4 overexpression 238 can up-regulate the expression of LMP2A and EBNA1, and knockdown CXCR4 can also reduce the expression of LMP2A and EBNA1. In conclusion, there is a positive feedback between CXCR4 and

240 EBV encoding products to influence the occurrence and development of EBVaGC, which is 241 regulated by some mechanism.

242 The results of Tang et al showed a new mechanism of NF-KB/NRF-1/CXCR4 circuit-mediated 243 TMP inhibition of neovascularization [27]. At the same time, many studies have shown that CXCR4 
244 can affect the occurrence and development of tumors through the NF-kB pathway, and the NFאB-

245 CXCR4 axis can also mediate the biological phenotype of tumors. By adding inhibitors of NF-kB

246 pathway into GT38 cells, we found that the expression of CXCR4 decreased with the increase of 247 inhibitor concentration, and the result was the same in SNU719. It was demonstrated that the NF-

$248 \kappa \mathrm{B}$ pathway activates CXCR4 in EBVaGC. In addition, CXCR4 binding to its ligand induces

249 conformational changes in itself that activate MAPK/ERK, PI3K/AKT, and JAK/STAT signaling

250 pathways [5, 8, 12, 19, 24, 26, 29]. Chang et al. found that CXCR4 activates phosphorylation of

$251 \mathrm{Ras} / \mathrm{Raf} / \mathrm{MEK} / \mathrm{ERK}$ pathway conjunctin Shc, thereby regulating cell cycle progression [3]. In this

252 study, we found that overexpression or knockdown of CXCR4 in EBVaGC cells increased or

253 decreased total ERK protein and phosphorylated ERK protein, confirming that the activation of ERK

254 signaling pathway is related to CXCR4 expression.

255 Our previous studies have demonstrated that LMP2A, the EBV coding product, induces NF-

$256 \kappa \mathrm{K}$ activation by regulating the expression of $\mathrm{I} \kappa \mathrm{B} \alpha$ and TRAF1. Structural activation of NF- $\mathrm{kB}$ is

257 involved in the malignant progression of EBVaGC by upregulation of genes involved in

258 proliferation, anti-apoptosis, and the maintenance of latent infection [35]. In this study, LMP2A

259 plasmid was transfected into EBV negative gastric cancer cells and LMP2A-siRNA was transfected

260 into EBV positive gastric cancer cells. We demonstrated that LMP2A could induce the activation of

261 NF- $\mathrm{kB}$. It was verified that the EBV coding product LMP2A activated CXCR4 through the NF- $\mathrm{BB}$

262 pathway. In order to further study the interaction between CXCR4 and EBV coding products in

263 EBVaGC, based on the activation of ERK signaling pathway by CXCR4, we added inhibitors of

264 ERK pathway in EBV positive cells, and found that the expression of LMP2A and EBNA1

265 decreased with the increase of inhibitor concentration, and the results were the same in SNU719. At

266 the same time, we also transfected ERK1/2-siRNAs into EBV positive gastric cancer cells, and the

267 result was the same as that of adding inhibitors. The results of Liu et al. showed that the double-

268 target knockout of miR-21 and CXCR4 could more effectively inhibit the proliferation, migration,

269 invasion and growth of glioma, and promote cell apoptosis, which may be related to PI3K/AKT and

270 Raf/MEK/ERK signaling pathways [14]. CXCR4-ERK signaling pathway mediates proliferation, 
migration, and invasion of cancer cells in multiple types of tumors $[2,15,18]$. In this study, EBV

272 positive cell SNU719-CXCR4 was transfected with CXCR4 plasmid and screened by G418, and

273 SNU719-CXCR4 cell line was successfully established. We found that after CXCR4 overexpression,

274 ERK pathway signal increased, EBV coding products LMP2A and EBNA1 were significantly and

275 stably increased, and the cell migration and proliferation ability of SNU719-CXCR4 group was

276 significantly higher than that of the control group, and the expression level of EMT-related proteins

277 was also significantly increased. In conclusion, EBV-encoded LMP2A activates CXCR4 through

278 the NF-kB pathway, and CXCR4 increases the expression of LMP2A and EBNA1 through the ERK

279 pathway, and affects related cell biological functions. LMP2A-NF-кB-CXCR4 and CXCR4-ERK-

280 LMP2A/EBNA1 may be potential therapeutic targets.

281 EBV has two life cycles: latent infection and lytic infection. During the latent infection, EBV

282 DNA exists in the nucleus of infected cells and only expresses a small amount of latent proteins

283 such as EBNA1 and LMP2A. After infection, EBV remains latent in the host cell, but to produce

284 the virus, it must induce the lytic phase of the virus cycle. [10]. BZLF1, an immediate early protein

285 of EBV, regulates the transition from latent infection to lytic infection. We found that BZLF1 was

286 significantly up-regulated in SNU719-NC cells with PKC activator (PMA/TPA) added. The

287 expression of BZLF1 was down-regulated in SNU719-CXCR4 cells. These results suggested that

288 CXCR4 was a negative regulator of BZLF1 expression. Meanwhile, the expression levels of LMP2A

289 and EBNA1 in SNU719-CXCR4 cells were significantly higher than those in SNU719-NC cells.

290 CXCR4 was confirmed to be involved in maintaining latent EBV infection by inhibiting BZLF1 and

291 subsequently inducing potential EBV products LMP2A and EBNA1.

292 In conclusion, there is a positive feedback between EBV latency coding products and CXCR4,

293 and LMP2A can activate CXCR4 through the NF-kB pathway. In addition, CXCR4 can be fed back

294 to LMP2A and EBNA1 through the ERK signaling pathway. Meanwhile, CXCR4 can reduce the

295 expression of the immediate early protein BZLF1, which plays an important role in maintaining the

296 latency infection of EBV. Therefore, the interaction of EBV coding products with CXCR4 is

297 important in the development of EBVaGC and provides a therapeutic option for successful 
treatment. However, the specific mechanisms need to be further studied to provide further reliable

299 basis for targeted therapy.

\section{Statements and Declarations}

The authors declare that they have no competing interests.

\section{Author contributions}

Bing Luo and Ni Qin conceived and designed the experiments; Ni Qin, Yan Zhang, Lin Xu, and Wen Liu performed the experiments; Ni Qin, Yan Zhang and Lin Xu analyzed the experimental data; Ni Qin drafted the manuscript; Ni Qin and Yan Zhang revised the manuscript. All authors reviewed and approved the final manuscript.

\section{Ethical approval and consent to participate}

We declare that this manuscript does not involve animal or human experiments, and does not involve informed consent.

\section{Acknowledgements}

This work was supported by Natural Science Foundation of Shandong Province [ZR2020MH302; ZR2020MC020]; China postdoctoral Science Foundation [2020M682126], and Qingdao Science and Technology Benefit the People Demonstration and Guidance Special Project (20-3-4-35-nsh)

\section{References:}

1. Bernhagen J, Krohn R, Lue H, Gregory JL, Zernecke A, Koenen RR, Dewor M, Georgiev I, Schober A, Leng L, Kooistra T, Fingerle-Rowson G, Ghezzi P, Kleemann R, McColl SR, Bucala R, Hickey MJ, Weber C (2007) MIF is a noncognate ligand of CXC chemokine receptors in inflammatory and atherogenic cell recruitment. Nature medicine 13:587-596. https://doi.org/10.1038/nm1567

2. Cai J, Zhang Q, Qian X, Li J, Qi Q, Sun R, Han J, Zhu X, Xie M, Guo X, Xia R (2020) Extracellular ubiquitin promotes hepatoma metastasis by mediating M2 macrophage polarization via the activation of the CXCR4/ERK signaling pathway. Annals of translational medicine 8:929. https://doi.org/10.21037/atm-20-1054

3. Chang F, Steelman LS, Shelton JG, Lee JT, Navolanic PM, Blalock WL, Franklin R, McCubrey JA (2003) Regulation of cell cycle progression and apoptosis by the Ras/Raf/MEK/ERK pathway (Review). International journal of oncology 22:469-480.

4. Chatterjee S, Behnam Azad B, Nimmagadda S (2014) The intricate role of CXCR4 in cancer. Advances in cancer research 124:31-82. https://doi.org/10.1016/b978-0-12- 
5. Gao H, Priebe W, Glod J, Banerjee D (2009) Activation of signal transducers and activators of transcription 3 and focal adhesion kinase by stromal cell-derived factor 1 is required for migration of human mesenchymal stem cells in response to tumor cell-conditioned medium. Stem cells (Dayton, Ohio) 27:857-865. https://doi.org/10.1002/stem.23

6. Hamm HE (1998) The many faces of G protein signaling. The Journal of biological chemistry 273:669-672. https://doi.org/10.1074/jbc.273.2.669

7. Hino R, Uozaki H, Inoue Y, Shintani Y, Ushiku T, Sakatani T, Takada K, Fukayama M (2008) Survival advantage of EBV-associated gastric carcinoma: survivin upregulation by viral latent membrane protein 2A. Cancer research 68:1427-1435. https://doi.org/10.1158/0008-5472.Can-07-3027

8. Huang CY, Lee CY, Chen MY, Yang WH, Chen YH, Chang CH, Hsu HC, Fong YC, Tang $\mathrm{CH}$ (2009) Stromal cell-derived factor-1/CXCR4 enhanced motility of human osteosarcoma cells involves MEK1/2, ERK and NF-kappaB-dependent pathways. Journal of cellular physiology 221:204-212. https://doi.org/10.1002/jcp.21846

9. Huo S, Luo Y, Deng R, Liu X, Wang J, Wang L, Zhang B, Wang F, Lu J, Li X (2020) EBV EBNA1 constructs an immunosuppressive microenvironment for nasopharyngeal carcinoma by promoting the chemoattraction of Treg cells. Journal for immunotherapy of cancer 8https://doi.org/10.1136/jitc-2020-001588

10. Kerr JR (2019) Epstein-Barr virus (EBV) reactivation and therapeutic inhibitors. Journal of clinical pathology 72:651-658. https://doi.org/10.1136/jclinpath-2019-205822

11. Khare T, Bissonnette M, Khare S (2021) CXCL12-CXCR4/CXCR7 Axis in Colorectal Cancer: Therapeutic Target in Preclinical and Clinical Studies. International journal of molecular sciences 22https://doi.org/10.3390/ijms22147371

12. Kufareva I, Salanga CL, Handel TM (2015) Chemokine and chemokine receptor structure and interactions: implications for therapeutic strategies. Immunology and cell biology 93:372-383. https://doi.org/10.1038/icb.2015.15

13. Li LN, Jiang KT, Tan P, Wang AH, Kong QY, Wang CY, Lu HR, Wang J (2015) Prognosis and Clinicopathology of CXCR4 in Colorectal Cancer Patients: a Meta-analysis. Asian Pacific journal of cancer prevention : APJCP 16:4077-4080. https://doi.org/10.7314/apjcp.2015.16.9.4077

14. Liu F, Yang B (2020) Double-Targeted Knockdown of miR-21 and CXCR4 Inhibits Malignant Glioma Progression by Suppression of the PI3K/AKT and Raf/MEK/ERK Pathways. BioMed research international 2020:7930160. https://doi.org/10.1155/2020/7930160

15. Liu Y, Wei S, Zou Q, Luo Y (2018) Stachydrine suppresses viability \& migration of astrocytoma cells via CXCR4/ERK \& CXCR4/Akt pathway activity. Future oncology (London, England) 14:1443-1459. https://doi.org/10.2217/fon-2017-0562 membrane protein 2. Trends in microbiology 4:38-42. https://doi.org/10.1016/0966- 
842x(96)81504-6

17. Luker KE, Luker GD (2006) Functions of CXCL12 and CXCR4 in breast cancer. Cancer letters 238:30-41. https://doi.org/10.1016/j.canlet.2005.06.021

18. Lv B, Yang X, Lv S, Wang L, Fan K, Shi R, Wang F, Song H, Ma X, Tan X, Xu K, Xie J, Wang G, Feng M, Zhang L (2015) CXCR4 Signaling Induced Epithelial-Mesenchymal Transition by PI3K/AKT and ERK Pathways in Glioblastoma. Molecular neurobiology 52:1263-1268. https://doi.org/10.1007/s12035-014-8935-y

19. Majka M, Ratajczak J, Kowalska MA, Ratajczak MZ (2000) Binding of stromal derived factor-1alpha (SDF-1alpha) to CXCR4 chemokine receptor in normal human megakaryoblasts but not in platelets induces phosphorylation of mitogen-activated protein kinase p42/44 (MAPK), ELK-1 transcription factor and serine/threonine kinase AKT. European journal of haematology 64:164-172. https://doi.org/10.1034/j.1600$\underline{0609.2000 .90112 . x}$

20. Murphy G, Pfeiffer R, Camargo MC, Rabkin CS (2009) Meta-analysis shows that prevalence of Epstein-Barr virus-positive gastric cancer differs based on sex and anatomic location. Gastroenterology 137:824-833. https://doi.org/10.1053/j.gastro.2009.05.001

21. Ribeiro J, Oliveira C, Malta M, Sousa H (2017) Epstein-Barr virus gene expression and latency pattern in gastric carcinomas: a systematic review. Future oncology (London, England) 13:567-579. https://doi.org/10.2217/fon-2016-0475

22. Saini V, Staren DM, Ziarek JJ, Nashaat ZN, Campbell EM, Volkman BF, Marchese A, Majetschak M (2011) The CXC chemokine receptor 4 ligands ubiquitin and stromal cell-derived factor- $1 \alpha$ function through distinct receptor interactions. The Journal of biological chemistry 286:33466-33477. https://doi.org/10.1074/jbc.M111.233742

23. Sleightholm RL, Neilsen BK, Li J, Steele MM, Singh RK, Hollingsworth MA, Oupicky D (2017) Emerging roles of the CXCL12/CXCR4 axis in pancreatic cancer progression and therapy. Pharmacology \& therapeutics 179:158-170. https://doi.org/10.1016/j.pharmthera.2017.05.012

24. Song ZY, Wang F, Cui SX, Qu XJ (2018) Knockdown of CXCR4 Inhibits CXCL12 Induced Angiogenesis in HUVECs through Downregulation of the MAPK/ERK and PI3K/AKT and the Wnt/ $\beta$-Catenin Pathways. Cancer investigation 36:10-18. https://doi.org/10.1080/07357907.2017.1422512

25. Speck SH, Chatila T, Flemington E (1997) Reactivation of Epstein-Barr virus: regulation and function of the BZLF1 gene. Trends in microbiology 5:399-405. https://doi.org/10.1016/s0966-842x(97)01129-3

26. Sun Y, Cheng Z, Ma L, Pei G (2002) Beta-arrestin2 is critically involved in CXCR4mediated chemotaxis, and this is mediated by its enhancement of p38 MAPK activation. The Journal of biological chemistry 277:49212-49219. https://doi.org/10.1074/jbc.M207294200

27. Tang M, Yang Y, Yu J, Qiu J, Chen P, Wu Y, Wang Q, Xu Z, Ge J, Yu K, Zhuang J (2018) Tetramethylpyrazine in a Murine Alkali-Burn Model Blocks NFkB/NRF-1/CXCR4- 

Signaling-Induced Corneal Neovascularization. Investigative ophthalmology \& visual science 59:2133-2141. https://doi.org/10.1167/iovs.17-23712

28. Tempera I, Klichinsky M, Lieberman PM (2011) EBV latency types adopt alternative chromatin conformations. PLoS pathogens 7:e1002180. https://doi.org/10.1371/journal.ppat.1002180

29. Vila-Coro AJ, Rodríguez-Frade JM, Martín De Ana A, Moreno-Ortíz MC, Martínez AC, Mellado M (1999) The chemokine SDF-1alpha triggers CXCR4 receptor dimerization and activates the JAK/STAT pathway. FASEB journal : official publication of the Federation of American Societies for Experimental Biology 13:1699-1710.

30. Wu B, Chien EY, Mol CD, Fenalti G, Liu W, Katritch V, Abagyan R, Brooun A, Wells P, Bi FC, Hamel DJ, Kuhn P, Handel TM, Cherezov V, Stevens RC (2010) Structures of the CXCR4 chemokine GPCR with small-molecule and cyclic peptide antagonists. Science (New York, NY) 330:1066-1071. https://doi.org/10.1126/science.1194396

31. Xiao J, Lai H, Wei SH, Ye ZS, Gong FS, Chen LC (2019) IncRNA HOTAIR promotes gastric cancer proliferation and metastasis via targeting miR-126 to active CXCR4 and RhoA signaling pathway. Cancer medicine 8:6768-6779. https://doi.org/10.1002/cam4.1302

32. Xu G, Lu K, Shen M, Zhang Q, Pan W, Tang Z (2020) Correlation between Chemokine CXCL-12 and ist Receptor CXCR4 Expression is Associated with Clinical Prognosis of Gastric Cancer. Clinical laboratory 66https://doi.org/10.7754/Clin.Lab.2019.190217

33. Xu J, Deng X, Tang M, Li L, Xiao L, Yang L, Zhong J, Bode AM, Dong Z, Tao Y, Cao Y (2013) Tyrosylprotein sulfotransferase- 1 and tyrosine sulfation of chemokine receptor 4 are induced by Epstein-Barr virus encoded latent membrane protein 1 and associated with the metastatic potential of human nasopharyngeal carcinoma. PloS one 8:e56114. https://doi.org/10.1371/journal.pone.0056114

34. Young LS, Murray PG (2003) Epstein-Barr virus and oncogenesis: from latent genes to tumours. Oncogene 22:5108-5121. https://doi.org/10.1038/sj.onc.1206556

35. Zhang Y, Liu W, Zhang W, Wang W, Song Y, Xiao H, Luo B (2019) Constitutive activation of the canonical NF-KB signaling pathway in EBV-associated gastric carcinoma. Virology 532:1-10. https://doi.org/10.1016/j.virol.2019.03.019

\section{Figure legends:}

Fig1: Interaction between LMP2A and CXCR4 at the expression level.

(a) Fluorescence efficiency of SNU719 and SGC7901 cells transfected with plasmids. Original magnification $\times 200$ for all panels. (b) Relative expression of LMP2A and CXCR4 were calculated using the comparative Cycle threshold $(\mathrm{Ct})$ value $\left(2^{-\Delta \Delta \mathrm{Ct}}\right)$ and actin as the internal standard. Data was represented as mean \pm standard error of mean (SEM) and came from at least three replicates. 
449 (c) The protein level of CXCR4 in SGC7901-LMP2A cell was detected by Western blot $\left({ }^{*} p<0.05\right)$.

450 (d) The effects on LMP2A and CXCR4 were analyzed by Western blot after interference of LMP2A 451 in GT38 cell line $\left({ }^{* *} p<0.01,{ }^{*} p<0.05\right.$, ns: no significant). (e) The protein level of EBNA1, LMP2A 452 and CXCR4 in SNU719-CXCR4 cell was detected by Western blot $\left(* * * p<0.001,{ }^{*} p<0.05\right)$. (f) The 453 effects on EBNA1, LMP2A and CXCR4 were analyzed by Western blot after interference of CXCR4 454 in SNU719 cell line $\left({ }^{* *} p<0.01,{ }^{*} p<0.05\right)$. Data are representative of three independent 455 experiments.

456 Fig2: LMP2A regulates CXCR4 expression through the NF-kB pathway.

457 (a) The protein level of $\mathrm{p} 65$ and p-p65 in SGC7901-LMP2A cell was detected by Western blot $(* * p$ $458<0.01$ ). (b) The effects on p65, p-p65 and LMP2A were analyzed by Western blot after interference

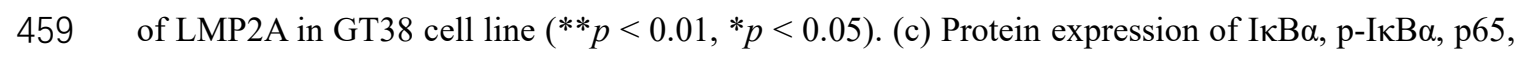
460 p-p65 and CXCR4 in GT38 after treated with $2 \mu \mathrm{M}$ and $4 \mu \mathrm{M}$ BAY -7085 for $24 \mathrm{~h}$ were analyzed by 461 Western blot. $\beta$-actin served as a control of loading. Densitometry values of the bands were expressed as fold-increases above the DMSO control (lane 1 and lane3) $\left({ }^{*} p<0.05\right.$, ns: no significant). (d) Protein expression of IкB $\alpha, \mathrm{p}-\mathrm{I} \kappa \mathrm{B} \alpha, \mathrm{p} 65$, p-p65 and CXCR4 in SNU719 after treated with $2 \mu \mathrm{M}$ and $4 \mu \mathrm{M}$ BAY -7085 for $24 \mathrm{~h}$ were analyzed by Western blotting. $\beta$-actin served as a control of loading. Densitometry values of the bands were expressed as fold-increases above the DMSO control (lane 1 and lane3) $\left({ }^{* *} p<0.01,{ }^{*} p<0.05\right.$, ns: no significant). Data are representative of three independent experiments.

Fig3: The effect of CXCR4 on ERK signal pathway.

(a) The protein level of ERK, p-ERK and CXCR4 in SNU719-CXCR4 cell was detected by Western blot $\left({ }^{*} p<0.05\right)$. (b) The effects on ERK, p-ERK and CXCR4 were analyzed by Western blot after interference of CXCR4 in GT38 cell line $\left({ }^{* *} p<0.01,{ }^{*} p<0.05\right)$. (c) The effects on ERK, p-ERK

472 and CXCR4 were analyzed by Western blot after interference of CXCR4 in SNU719 cell line $(* * p$ $\left.473<0.01,{ }^{*} p<0.05\right)$. Data are representative of three independent experiments.

474 Fig4: The effect of ERK signaling on LMP2A and EBNA1 expression.

475 (a) Protein expression of ERK, p-ERK, LMP2A and EBNA1 in GT38 after treated with $2 \mu \mathrm{M}$ and 4 
$476 \mu \mathrm{M}$ PD0325901 for $48 \mathrm{~h}$ were analyzed by western blotting. $\beta$-actin served as a control of loading.

477 Densitometry values of the bands were expressed as fold-increases above the DMSO control (lane

4781 and lane3) $\left({ }^{* *} p<0.001,{ }^{* *} p<0.01,{ }^{*} p<0.05\right.$, ns: no significant). (b) Protein expression of ERK, 479 p-ERK, LMP2A and EBNA1 in SNU719 after treated with $2 \mu \mathrm{M}$ and $4 \mu \mathrm{M}$ PD0325901 for $48 \mathrm{~h}$ 480 were analyzed by Western blot. $\beta$-actin served as a control of loading. Densitometry values of the 481 bands were expressed as fold-increases above the DMSO control (lane 1 and lane3) $482\left(* * * p<0.001,{ }^{* *} p<0.01,{ }^{*} p<0.05\right)$. (c) The effects on ERK, p-ERK, LMP2A and EBNA1 were 483 analyzed by Western blot after interference of ERK in GT38 cell line $\left({ }^{* *} p<0.01,{ }^{*} p<0.05\right.$, ns: no 484 significant). (d) The effects on ERK, p-ERK, LMP2A and EBNA1 were analyzed by Western blot 485 after interference of ERK in SNU719 cell line $\left({ }^{*} p<0.01,{ }^{*} p<0.05\right.$, ns: no significant). Data are 486 representative of three independent experiments.

487 Fig5: Effect of CXCR4 on cell migration and proliferation and maintenance of EBV latent 488 infection.

489 (a) The role of CXCR4 on cell migration were assessed by transwell migration assay at $24 \mathrm{~h}$ under 490 the microscope (200x). (b) The proliferative capacity of CXCR4 was evaluated by CCK-8 assay in 491 SNU719-CXCR4 cell $\left(* * p<0.01,{ }^{*} p<0.05\right)$. (c) The protein level of N-cadherin, E-cadherin, 492 MMP-2, Vimentin and CXCR4 in SNU719-CXCR4 cell was detected by Western blot $(* * p<0.01$, $493 * p<0.05$ ). Data are representative of three independent experiments. (d) Western blotting was used 494 to compare BZLF1, CXCR4, LMP2A and EBNA1 protein expression in the DMSO, 495 DMSO+transfected CXCR4,TPA and TPA+transfected CXCR4 groups. The averages of three 496 independent replicates are shown.

497 Fig6: Graphic summary.

498 EBV encoded latent membrane protein 2A activates seven transmembrane proteins CXCR4 through 499 the NF- $\mathrm{BB}$ signaling pathway, and CXCR4 upregulates EBV encoded latent membrane protein 2A 500 and EBV nuclear antigen 1 through the ERK signaling pathway. 


\section{Figures}

$a^{-}$
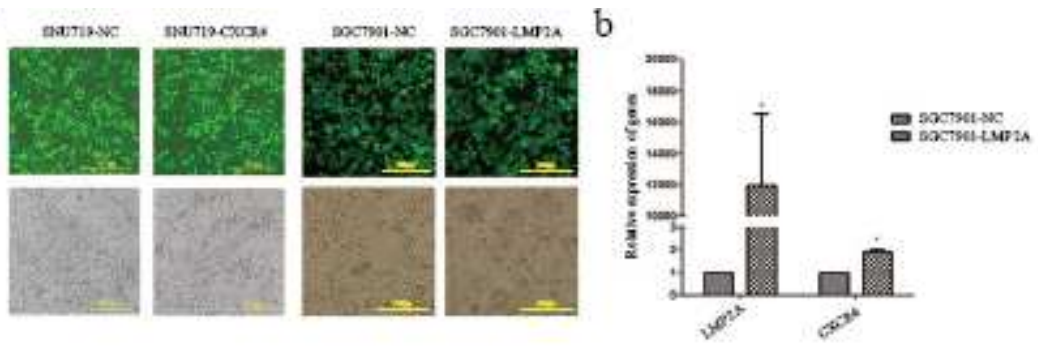

c

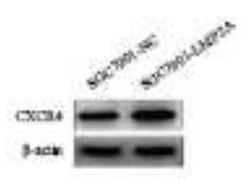

d

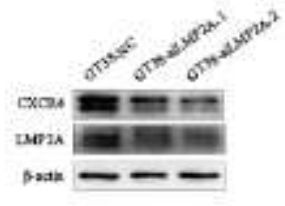

$\mathrm{e}$

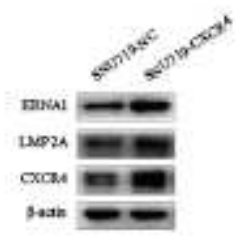

f

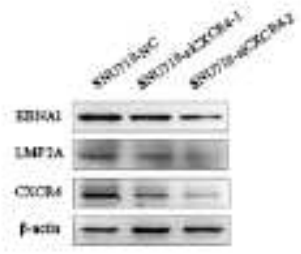

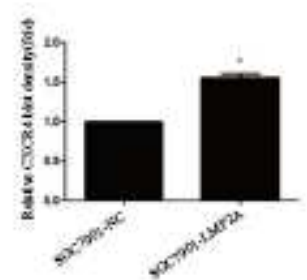
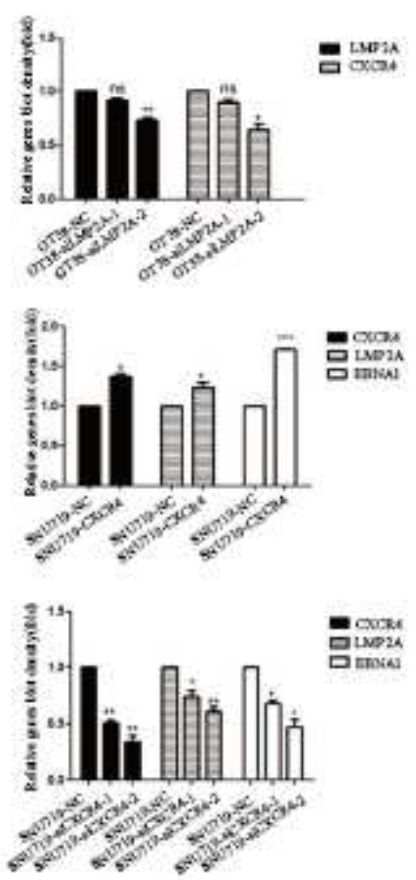

\section{Figure 1}

Interaction between LMP2A and CXCR4 at the expression level. (a) Fluorescence efficiency of SNU719 and SGC7901 cells transfected with plasmids. Original magnification $\times 200$ for all panels. (b) Relative expression of LMP2A and CXCR4 were calculated using the comparative Cycle threshold (Ct) value (2- $\triangle \Delta \mathrm{Ct})$ and actin as the internal standard. Data was represented as mean \pm standard error of mean (SEM) and came from at least three replicates. (c) The protein level of CXCR4 in SGC7901-LMP2A cell was detected by Western blot ( ${ }^{*} p<0.05$ ). (d) The effects on LMP2A and CXCR4 were analyzed by Western blot after interference of LMP2A in GT38 cell line (** $p<0.01,{ }^{\star} p<0.05$, ns: no significant). (e) The protein level of EBNA1, LMP2A and CXCR4 in SNU719-CXCR4 cell was detected by Western blot $\left(\star \star \star p<0.001,{ }^{*} p<0.05\right)$. (f) The effects on EBNA1, LMP2A and CXCR4 were analyzed by Western blot after 
interference of CXCR4 in SNU719 cell line $\left(* \star p<0.01,{ }^{\star} p<0.05\right)$. Data are representative of three independent experiments.

a

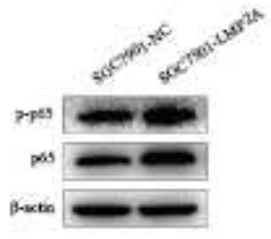

b

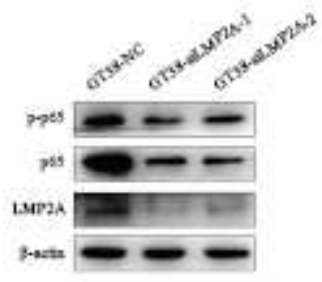

$\mathrm{C}$

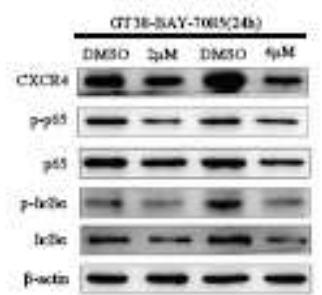

d

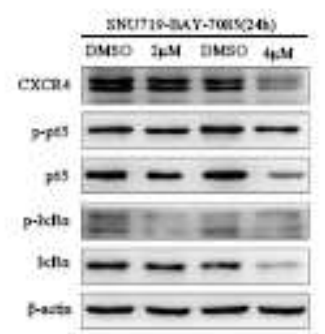

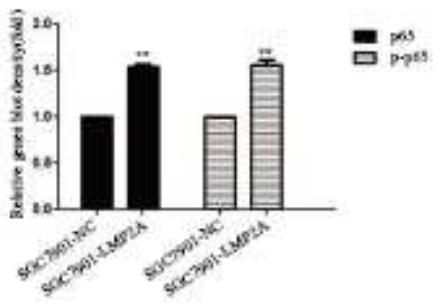
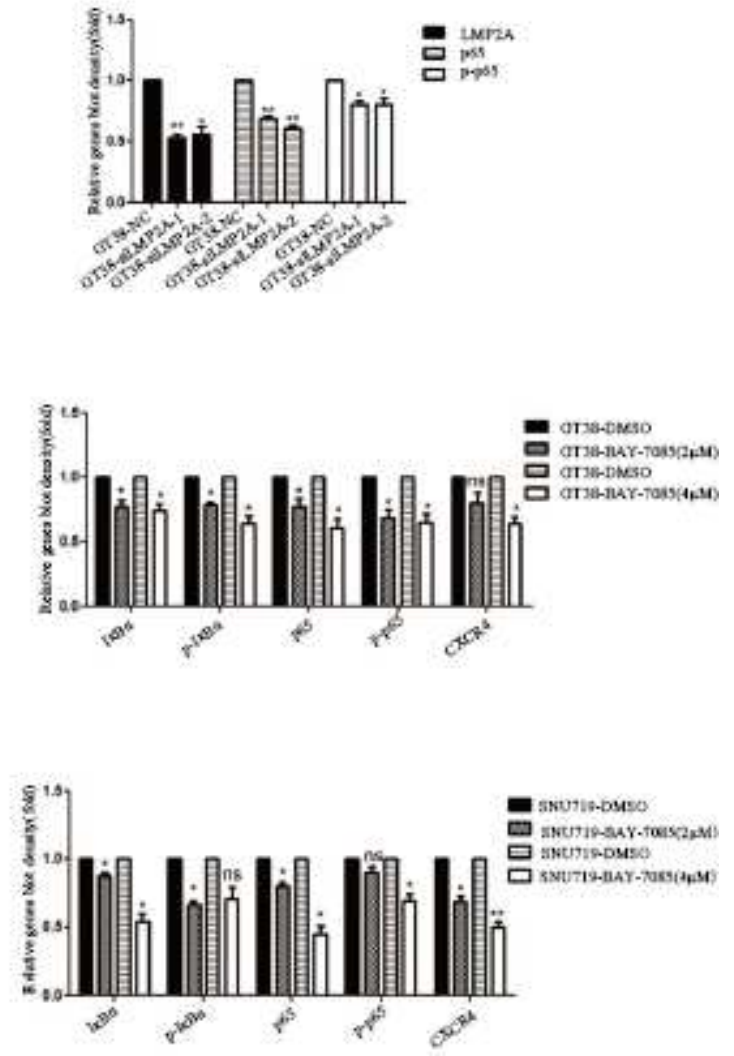

Figure 2

LMP2A regulates CXCR4 expression through the NF-KB pathway. (a) The protein level of p65 and p-p65 in SGC7901-LMP2A cell was detected by Western blot $(* \star p<0.01)$. (b) The effects on p65, p-p65 and LMP2A were analyzed by Western blot after interference of LMP2A in GT38 cell line ( ${ }^{\star \star} p<0.01$, ${ }^{\star} p<$ 0.05). (c) Protein expression of IKBa, p-IкBa, p65, p-p65 and CXCR4 in GT38 after treated with $2 \mu \mu \mathrm{M}$ and $4 \mu \mathrm{M}$ BAY -7085 for $24 \mathrm{~h}$ were analyzed by Western blot. $\beta$-actin served as a control of loading. Densitometry values of the bands were expressed as fold-increases above the DMSO control (lane 1 and lane3) (*p < 0.05, ns: no significant). (d) Protein expression of ІкBa, p-ІкBa, p65, p-p65 and CXCR4 in SNU719 after treated with $2 \mu \mu \mathrm{M}$ and $4 \mu \mathrm{M}$ BAY -7085 for $24 \mathrm{~h}$ were analyzed by Western blotting. $\beta$-actin served as a control of loading. Densitometry values of the bands were expressed as fold-increases above the DMSO control (lane 1 and lane3) $\left({ }^{\star \star} \mathrm{p}<0.01,{ }^{\star} \mathrm{p}<0.05\right.$, ns: no significant). Data are representative of three independent experiments. 
a

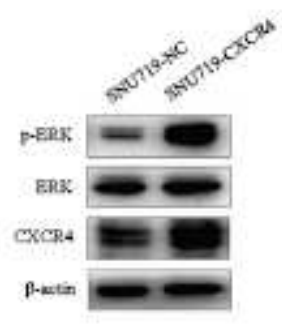

b

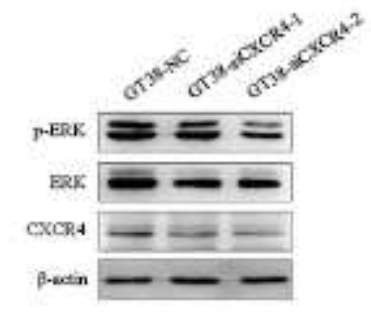

c

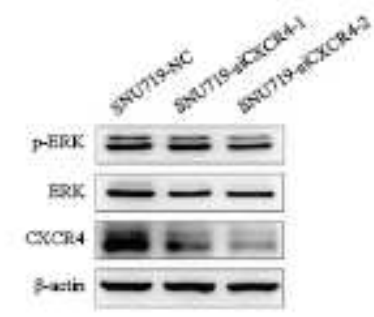

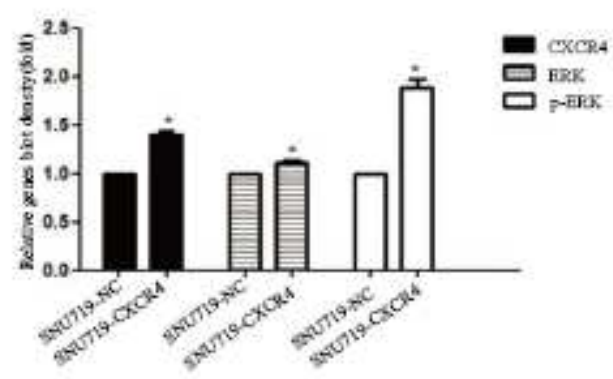

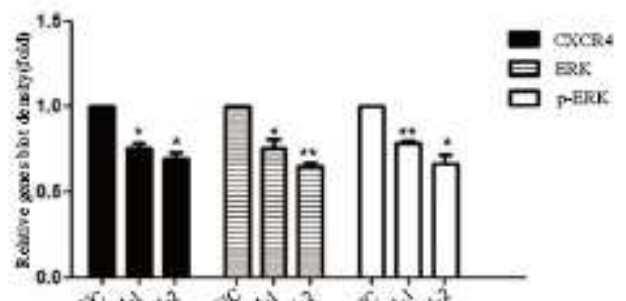

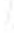

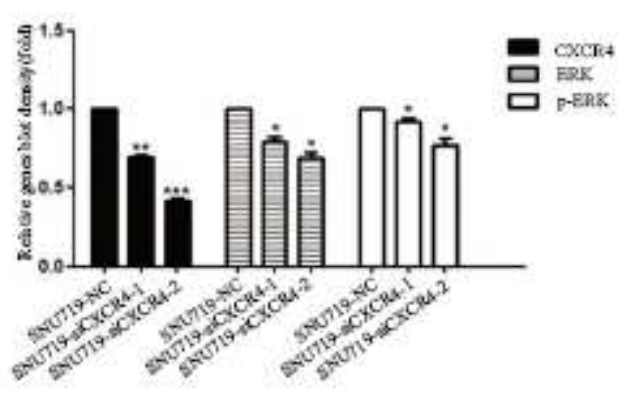

Figure 3

The effect of CXCR4 on ERK signal pathway. (a) The protein level of ERK, p-ERK and CXCR4 in SNU719CXCR4 cell was detected by Western blot $\left({ }^{*} p<0.05\right)$. (b) The effects on ERK, $p$-ERK and CXCR4 were analyzed by Western blot after interference of CXCR4 in GT38 cell line $\left({ }^{\star} p<<0.01,{ }^{*} p<0.05\right)$. (c) The effects on ERK, p-ERK and CXCR4 were analyzed by Western blot after interference of CXCR4 in SNU719 cell line $\left({ }^{*} p<0.01,{ }^{\star} p<0.05\right)$. Data are representative of three independent experiments. 

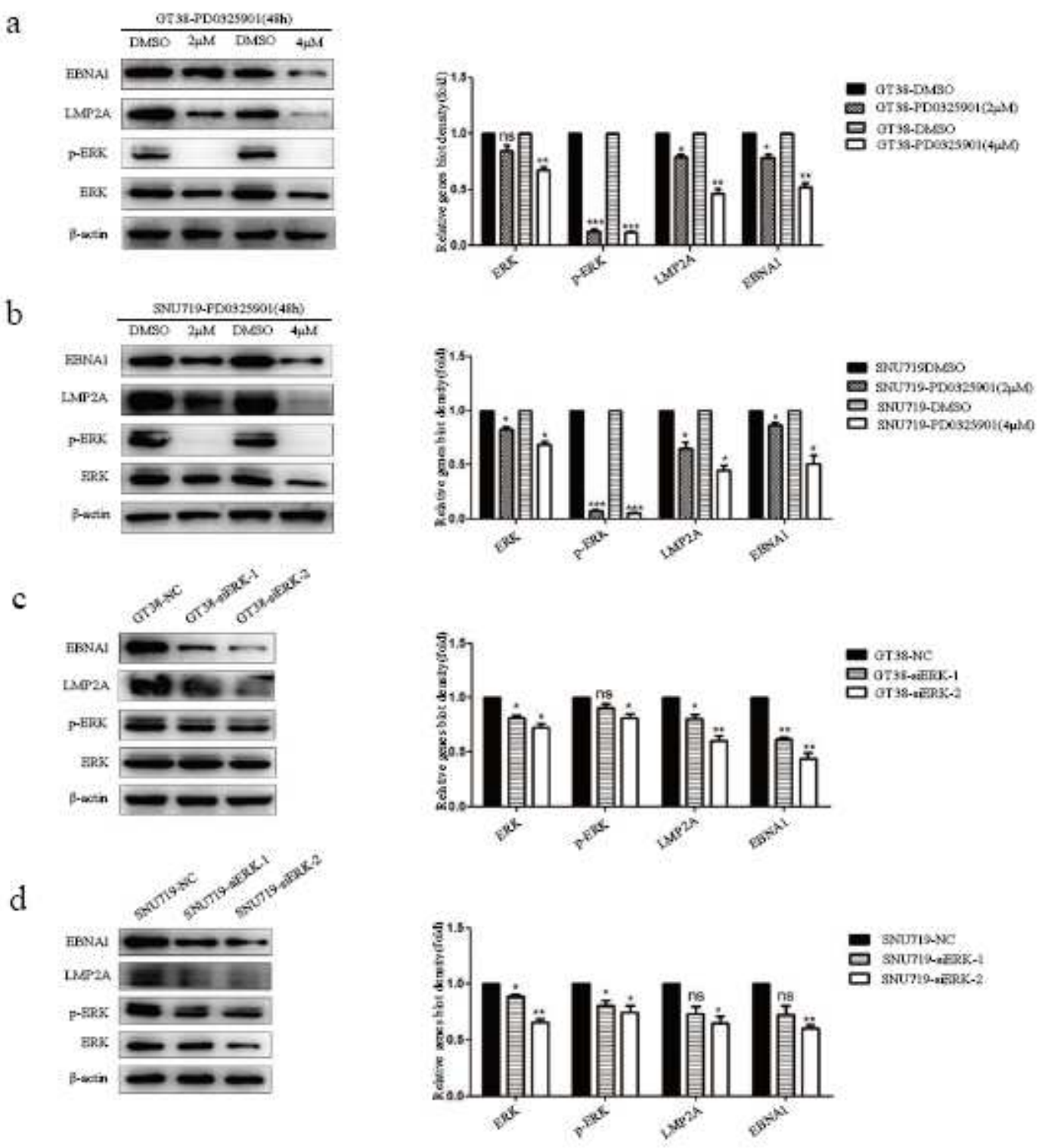

Figure 4

The effect of ERK signaling on LMP2A and EBNA1expression. (a) Protein expression of ERK, p-ERK, LMP2A and EBNA1 in GT38 after treated with $2 \mu \mu \mathrm{M}$ and $4 \mu \mathrm{M}$ PD0325901 for $48 \mathrm{~h}$ were analyzed by western blotting. $\beta$-actin served as a control of loading. Densitometry values of the bands were expressed as fold-increases above the DMSO control (lane 1 and lane3) (** $<<0.001$, $* \star p p<0.01$, ${ }^{\star} p<0.05$, ns: no significant). (b) Protein expression of ERK, p-ERK, LMP2A and EBNA1 in SNU719 after treated with $2 \mu \mu \mathrm{M}$ and $4 \mu \mathrm{M}$ PD0325901 for $48 \mathrm{~h}$ were analyzed by Western blot. $\beta$-actin served as a control of loading. Densitometry values of the bands were expressed as fold-increases above the DMSO control (lane 1 and lane3) $\left(\star \star \star p<0.001,{ }^{\star \star} p<0.01,{ }^{\star} p<0.05\right)$. (c) The effects on ERK, p-ERK, LMP2A and EBNA1 were analyzed by Western blot after interference of ERK in GT38 cell line ( ${ }^{\star \star} p<0.01$, ${ }^{\star} p<0.05$, ns: no significant). (d) The effects on ERK, p-ERK, LMP2A and EBNA1 were analyzed by Western blot after interference of ERK in SNU719 cell line ( ${ }^{\star \star} p<0.01,{ }^{\star} p<0.05$, ns: no significant). Data are representative of three independent experiments. 
a
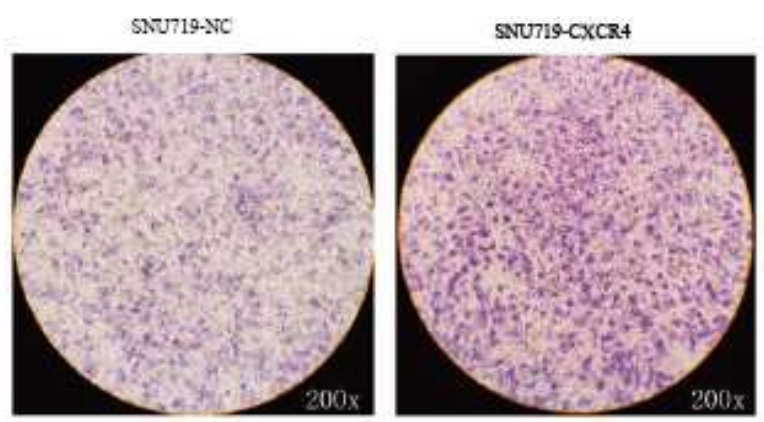

b

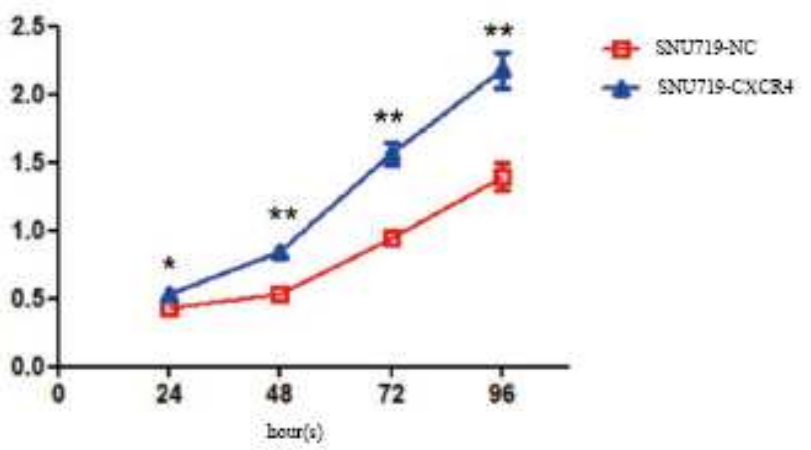

C

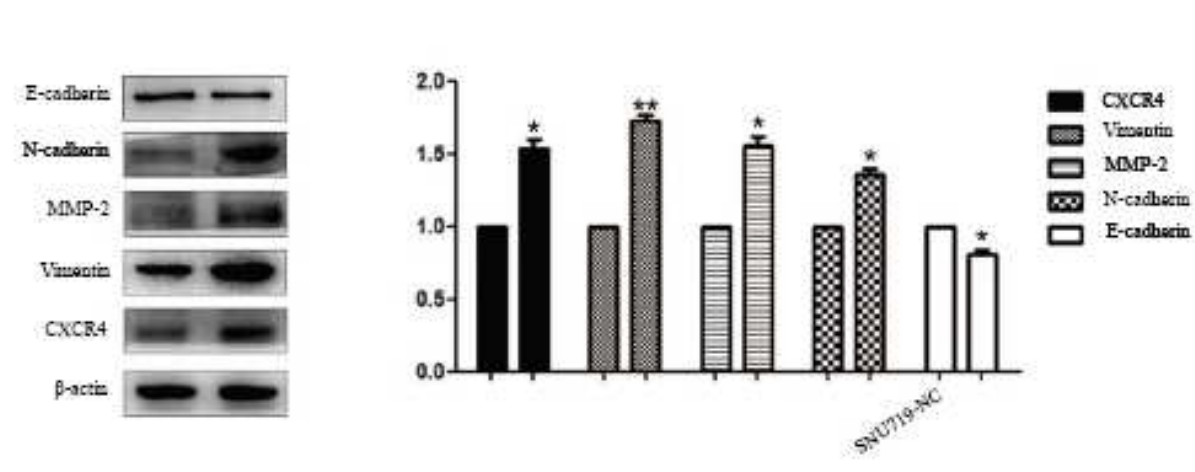

$\mathrm{d}$

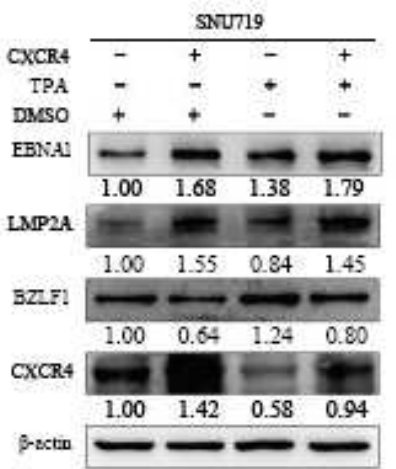

Figure 5

Effect of CXCR4 on cell migration and proliferation and maintenance of EBV latent infection. (a) The role of CXCR4 on cell migration were assessed by transwell migration assay at $24 \mathrm{~h}$ under the microscope (200x). (b) The proliferative capacity of CXCR4 was evaluated by CCK-8 assay in SNU719-CXCR4 cell (**p $<0.01,{ }^{*} \mathrm{p}<0.05$ ). (c) The protein level of N-cadherin, E-cadherin, MMP-2, Vimentin and CXCR4 in SNU719CXCR4 cell was detected by Western blot $\left(* * p<0.01,{ }^{*} p<0.05\right)$. Data are representative of three independent experiments. (d) Western blotting was used to compare BZLF1, CXCR4, LMP2A and EBNA1 protein expression in the DMSO, DMSO+transfected CXCR4,TPA and TPA+transfected CXCR4 groups. The averages of three 495 independent replicates are shown. 


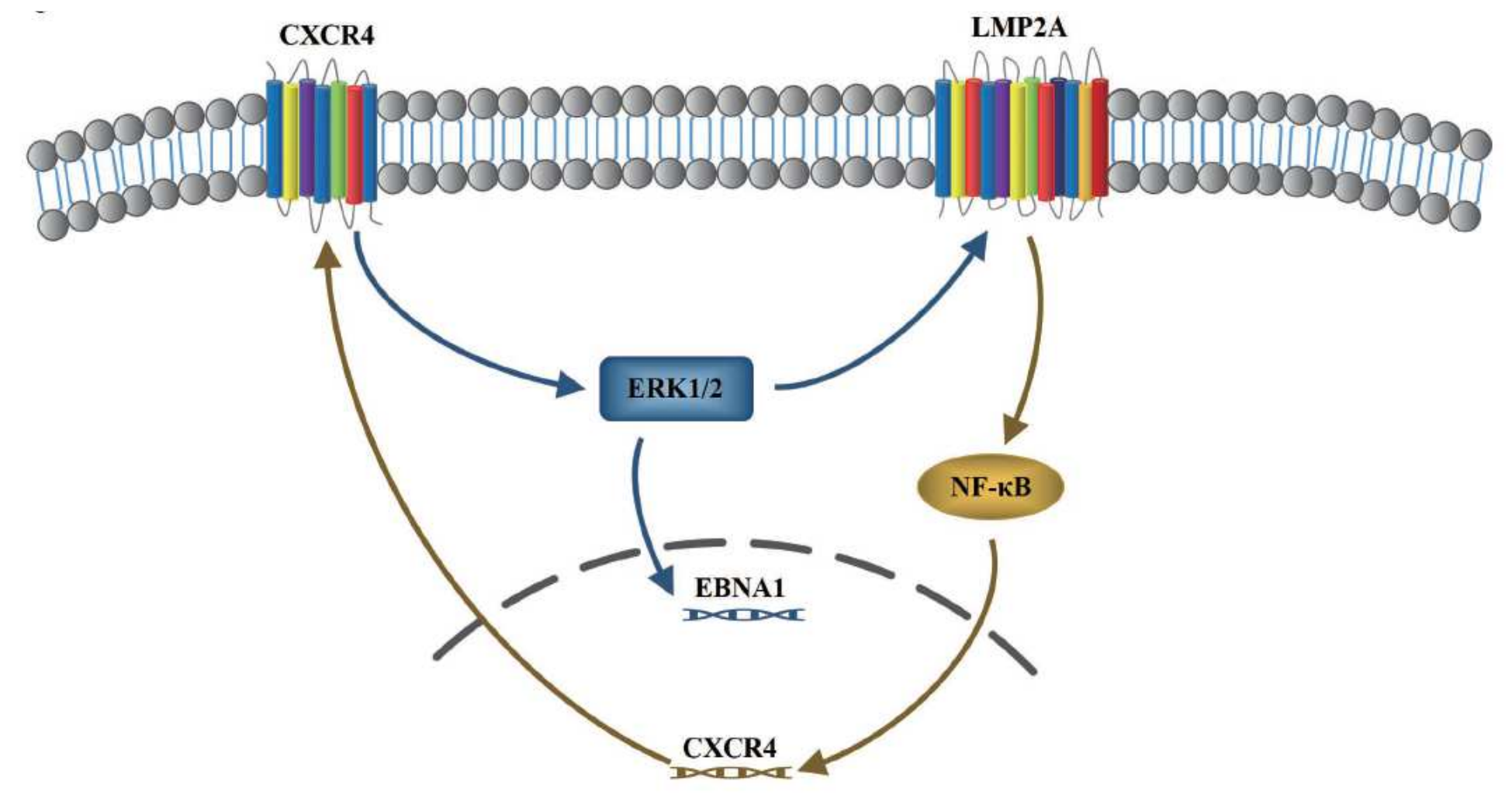

Figure 6

Graphic summary. EBV encoded latent membrane protein 2A activates seven transmembrane proteins CXCR4 through the NF-KB signaling pathway, and CXCR4 upregulates EBV encoded latent membrane protein $2 \mathrm{~A}$ and EBV nuclear antigen 1 through the ERK signaling pathway. 